\title{
Liter per Minute per Milligram per Kilogram per Day
}

National Cancer Institute

\section{Source}

National Cancer Institute. Liter per Minute per Milligram per Kilogram per Day. NCI

Thesaurus. Code C120753.

A unit of concentration equal to liter per minute divided by milligram per kilogram per day. 